\title{
Phase diagram and topological phases in the triangular lattice Kitaev-Hubbard model
}

\author{
Kai Li, Shun-Li Yu, Zhao-Long Gu, and Jian-Xin Li* \\ National Laboratory of Solid State Microstructures and Department of Physics, Nanjing University, Nanjing 210093, China \\ Collaborative Innovation Center of Advanced Microstructures, Nanjing University, Nanjing, China
}

(Dated: September 15, 2016)

\begin{abstract}
We study the half-filled Hubbard model on the triangular lattice with spin-dependent Kitaevlike hopping. Using the variational cluster approach, we identify five phases: a metallic phase, a non-coplanar chiral magnetic order, a $120^{\circ}$ magnetic order, a nonmagnetic insulator (NMI), and an interacting Chern insulator (CI) with a nonzero Chern number. The transition from CI to NMI is characterized by the change of the charge gap from an indirect band gap to a direct Mott gap. Based on the slave-rotor mean-field theory, the NMI phase is further suggested to be a gapless Mott insulator with a spinon Fermi surface or a fractionalized CI with nontrivial spinon topology, depending on the strength of Kitaev-like hopping. Our work highlights the rising field that interesting phases emerge from the interplay of band topology and Mott physics.
\end{abstract}

PACS numbers:

\section{INTRODUCTION}

Emergent quantum phenomena arising from the interplay of band topology and electron correlation are currently under intense investigation ${ }^{1,2}$. Following the discovery of topological insulators ${ }^{3-5}$, it is now recognized that spin-orbit coupling (SOC) is an essential ingredient for the emergence of nontrivial band topology. In parallel, correlated electron physics is a venerable but still vibrant subject. A body of phenomena arises from this subject, including quantum magnetism, high-temperature superconductivity, and factional quantum Hall effect ${ }^{6-8}$. Strong SOC and electron correlation come together in the heavy transition metal compounds such as $5 d$ series $^{9-11}$, and interesting physics arises ${ }^{1,12-14}$. For example, it was demonstrated by Jackeli and Khaliullin ${ }^{10}$ that in a class of late transition metal oxides with an edge-shared octahedral structure, strong SOC together with electron correlation would lead the interactions between spin-orbit entangled effective $J_{\text {eff }}=1 / 2$ moments to be highly anisotropic. The associated low energy effective Hamiltonian for $J_{\text {eff }}$ is the Kitaev-Heisenberg model ${ }^{15-19}$, which hosts a finite window of Kitaev spin-liquid phase ${ }^{20}$. This model has also been suggested to describe the zigzag and spiral magnetic orders observed in iridates $A_{2} \operatorname{IrO}_{3}(A=$ $\mathrm{Na}, \mathrm{Li})^{21,22}$.

An essential feature of SOC is that it entangles the spin and spatial degrees of freedom of electrons. In fact, similar effects can be realized by considering a type of bond-selective and spin-dependent Kitaev-like hoppings. They take the form $t^{\prime} c_{i}^{\dagger} \tau^{\alpha} c_{j}$, where $c_{i}^{\dagger}=\left(c_{i \uparrow}^{\dagger}, c_{i \downarrow}^{\dagger}\right)$ creates an electron at site $i$ and the Pauli matrices $\tau^{\alpha}(\alpha=x, y, z)$ depend on the hopping directions. Such a nearestneighbor Kitaev-like hopping on the honeycomb lattice was originally proposed as a way of realizing the Kitaev spin model ${ }^{20}$ in cold atom systems ${ }^{23}$. A next-nearestneighbor Kitaev-like hopping on the honeycomb lattice plays a similar role as the intrinsic SOC in the KaneMele $\operatorname{model}^{3}$, in the sense of giving rise to a nontrivial band topology. This Kitaev-like hopping also appears in an effective tight-binding model for $\mathrm{Na}_{2} \mathrm{IrO}_{3}$ via firstprinciples calculations ${ }^{24}$.

The incorporation of Kitaev-like hopping and electron correlation is thus expected to lead to other novel quantum phases. A recent numerical study of the KitaevHubbard model on a bipartite honeycomb lattice suggests an algebraic spin-liquid phase ${ }^{25}$. This model is basically a Hubbard model with Kitaev-like hopping, and its effective spin model in the large- $U$ limit is the Kitaev-Heisenberg model, thereby dubbed as the Kitaev-Hubbard model ${ }^{25,26}$. The algebraic spin-liquid phase in this model is a time-reversal (TR) symmetric nonmagnetic insulator (NMI) with gapless spinon excitations $^{25}$, which intimately relates to the gapless Kitaev spin liquid ${ }^{20}$. The TR symmetry due to the bipartite nature of honeycomb lattice protects the gaplessness of these spin-liquid phases. By contrast, a non-bipartite lattice structure with geometric frustration may break TR symmetry and open a gap to the spinon spectrum, endowing a NMI phase with nontrivial topology $25,27,28$.

Motivated by these studies, it is instructive to consider the Kitaev-Hubbard model defined on a non-bipartite triangular lattice to look for what novel quantum phases would emerge. At large- $U$ limit, this model is described effectively by the triangular Kitaev-Heisenberg model. So, another motivation comes from the very recent experimental investigations on $\mathrm{YbMgGaO}_{4}{ }^{29-32}$ and $\mathrm{Ba}_{3} \mathrm{IrTi}_{2} \mathrm{O}_{9}{ }^{33}$, which are triangular lattice materials with strong SOC and host possible spin liquid ground states. We note that the proposed spin Hamiltonian ${ }^{29}$ for $\mathrm{YbMgGaO}_{4}$ can be reduced to the triangular Kitaev-Heisenberg model for a special set of coupling constants ${ }^{34}$, and the low-energy effective isospin $J_{\text {eff }}$ Hamiltonian for $\mathrm{Ba}_{3} \mathrm{IrTi}_{2} \mathrm{O}_{9}$ was suggested ${ }^{11}$ to be the triangular Kitaev-Heisenberg model.

In this paper, we study the quantum phases and quantum phase transitions in the half-filled triangular lattice Hubbard model with Kitaev-like hopping $t^{\prime}$. In the noninteracting case, the system undergoes a metal-Chern insulator(CI) transition at $t_{c}^{\prime}=\sqrt{3} t$ with increasing $t^{\prime}$ 
( $t$ the usual spin-conserving hopping). This transition arises from the appearance of an indirect energy gap between two bands with nonzero Chern numbers +2 and -2 due to $t^{\prime}$. Using the variational cluster approach (VCA), we obtain the $\left(t^{\prime}, U\right)$ ( $U$ the Hubbard interaction) phase diagram. In addition to the metallic phase and $120^{\circ}$ antiferromagnetic insulator (AFI), we observe three more phases: a non-coplanar chiral spin density wave (SDW), an interacting CI, and a NMI. Inside the metal phase, the chiral SDW appears as a weak-coupling instability in a limited window of $t^{\prime}$ at which the Fermi level approaches the van Hove singularities. The CI phase with a nonzero Chern number survives a wide region of Hubbard interaction $U$ up to $U \sim 13.5 t$, and hence an interacting CI is obtained. We find that the critical value $t_{c}^{\prime}$ does not change with $U$, so a straight line with $t_{c}^{\prime}=\sqrt{3} t$ separates the metal and interacting CI phases. The NMI refers to the phase with a finite single-particle gap but no long-range magnetic order. For $t^{\prime}<t_{c}^{\prime}$, the metal to NMI transition is characterized by the opening of singleparticle gap with increasing $U$. While for $t^{\prime}>t_{c}^{\prime}$, the transition from CI to NMI is accompanied by the change of charge gap from an indirect band gap to a direct Mott gap. Using the slave-rotor approach, the NMI phase is further predicted to be a gapless Mott insulator with a spinon Fermi surface for $t^{\prime}<t_{c}^{\prime}$ or a fractionalized CI with bulk gapped spinon excitations for $t^{\prime}>t_{c}^{\prime}$. The spinons in fractionalized CI inherit the nontrivial band topology of the noninteracting CI, as expected from the above discussions of non-bipartite lattice structure and nontrivial topology in the presence of Kitaev-like hopping $t^{\prime}$.

This paper is organized as follows. In Sec. II we introduce our model and summarize the numerical approach. In Sec. III we discuss our numerical results for the phase diagram. Sec. IV presents a slave-rotor analysis of our model. Sec. V gives the summary and discussion. Detailed discussions of the band topology and mean-field calculations can be found in appendices.

\section{MODEL AND METHOD}

The triangular lattice Kitaev-Hubbard model is defined by the Hamiltonian

$$
\begin{aligned}
H & =H_{0}+U \sum_{i} \hat{n}_{i \uparrow} \hat{n}_{i \downarrow} \\
H_{0} & =-\sum_{\langle i, j\rangle} c_{i}^{\dagger}\left(t \tau^{0}+t^{\prime} \tau^{\alpha}\right) c_{j}-\mu \sum_{i, \sigma} \hat{n}_{i \sigma}
\end{aligned}
$$

where $c_{i}^{\dagger}=\left(c_{i \uparrow}^{\dagger}, c_{i \downarrow}^{\dagger}\right), c_{i \sigma}^{\dagger}$ creates an electron at site $i$ with spin $\sigma$, and $\hat{n}_{i \sigma}=c_{i \sigma}^{\dagger} c_{i \sigma}$. The term with the identity matrix $\tau^{0}$ is the usual spin-conserving hopping. The term with bond-dependent Pauli matrices $\tau^{\alpha}(\alpha=x, y, z)$ as illustrated in Fig.1(a) and a real hopping amplitude $t^{\prime}$ represents the Kitaev-like hopping, which breaks TR symmetry and spin-rotation symmetry.
In the noninteracting limit $(U=0)$, the Kitaev-like hopping $t^{\prime}$ endows each band with a nonzero Chern number +2 or -2 , which corresponds to two gapless chiral edge modes shown in Fig.1(d). At half filling, this leads to a CI for $t^{\prime}>\sqrt{3} t$, and a metal exists for $t^{\prime}<\sqrt{3} t$ [see Appendix A 1]. There is thus a metal-CI transition at $t^{\prime}=\sqrt{3} t$. Here, the Chern number \pm 2 can be interpreted by the contributions from four Dirac points that arise from the smoothly deformed Bloch Hamiltonian, as shown in Appendix A 2. We also give a general constraint on even integer valued Chern numbers in a class of Bloch Hamiltonians in Appendix A 3.
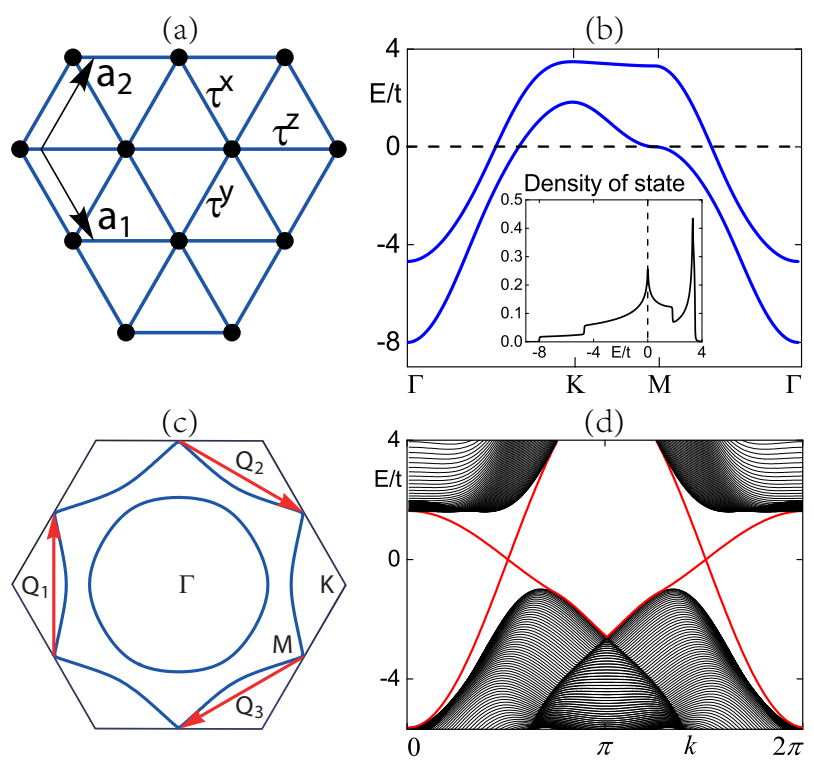

FIG. 1: (color online). (a) 12-site cluster tiling the triangular lattice used in VCA calculations. The Kitaev-like hopping bonds are indicated by Pauli matrices $\tau^{\alpha} . \boldsymbol{a}_{1}$ and $\boldsymbol{a}_{2}$ are lattice primitive vectors. (b) The energy dispersion and density of state (inset) at $t^{\prime} \simeq 0.478 t, U=0$. The dashed line denotes Fermi level. (c) At $t^{\prime} \simeq 0.478 t$ and $U=0$, the Fermi-surface (blue line) crosses Brillouin zone boundary at six van Hove singularity points ( $M$ points). (d) The single-particle spectrum for a cylindrical geometry of triangular lattice shows the chiral edge states (red lines), calculated at $t^{\prime}=2.3 t, U=0$.

In the interacting case, we study the model using cluster perturbation theory $(\mathrm{CPT})^{35}$ and $\mathrm{VCA}^{36}$, both of which have been successfully applied to the study of strongly correlated systems ${ }^{12,37-40}$. CPT proceeds by dividing the lattice into a superlattice of identical clusters. The cluster single-particle Green's function $G_{c}$ is calculated by exact diagonalization and the inter-cluster hopping terms $V$ are treated perturbatively. So, the Green's function $G$ for the whole system can be obtained via $G^{-1}=G_{c}^{-1}-V$. In this method, the tiling of the lattice into identical clusters makes up a reference system with the same two-body on-site interaction as the original system but a different one-body part due to the approximate treatment of inter-cluster hoppings. Because the solutions of the clusters are exact, the short-range (within 
each cluster) correlations have been taken into account. Therefore, we can expect that this method provides a good approximation for correlated systems such as the Hubbard model where short-range correlations dominate the physics. VCA is an extension of CPT. It is used to explore symmetry-breaking phases, in which the grand potential $\Omega(h)$ as a function of the symmetry-breaking Weiss field $h$ can be obtained. The corresponding phase exists once we have $\partial \Omega(h) / \partial h=0$. In our calculations, we will use the 12-site cluster shown in Fig.1(a). This is the largest available cluster considering that: (1) it preserves the 3 -fold rotation symmetry and hence treats the three kinds of Kitaev-like hopping terms on the same footing; (2) it consists of multiples of four (three) sites for the chiral SDW $\left(120^{\circ}\right.$ AFI) order; and (3) it has even number of sites so that the NMI phase can be hosted.

In the presence of interactions, the Chern number is calculated via the single-particle Green's function $G^{41-44}$. As has been shown recently ${ }^{44,45}$, one can solve the eigenvalue equation $h(\boldsymbol{k})|\boldsymbol{k}, n\rangle=\varepsilon_{n}(\boldsymbol{k})|\boldsymbol{k}, n\rangle$ with $h(\boldsymbol{k}) \equiv$ $-G^{-1}(i \omega=0, \boldsymbol{k})$, and then calculate the Chern number via

$C=\frac{1}{2 \pi i} \int d k_{1} d k_{2} \sum_{\varepsilon_{n}<0, \varepsilon_{m}>0} \frac{\left\langle n\left|\partial_{k_{1}} h\right| m\right\rangle\left\langle m\left|\partial_{k_{2}} h\right| n\right\rangle-H . c .}{\left(\varepsilon_{n}-\varepsilon_{m}\right)^{2}}$.

\section{NUMERICAL RESULTS}

Our main results obtained via CPT and VCA are summarized in the phase diagram shown in Fig.2.

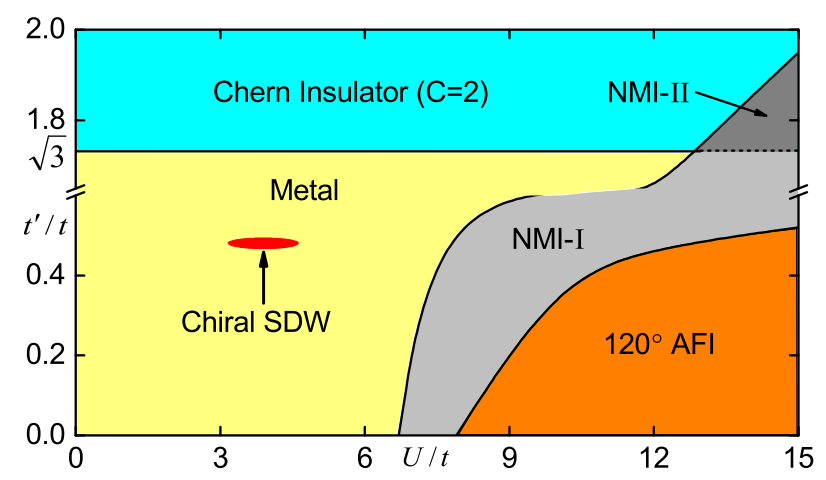

FIG. 2: (color online). Phase diagram obtained by VCA. SDW, AFI, and NMI denote the spin density wave, antiferromagnetic insulator and nonmagnetic insulator, respectively. The NMI phase will be further divided into the NMI-I and -II phases which denote the gapless Mott insulator and the fractionalized Chern insulator suggested by the slave-rotor theory.

Inside the metallic region, we find a non-coplanar chiral SDW phase, which was previously observed in the context of spin models with two- and four-spin exchange interactions ${ }^{46,47}$. Here, the motivation to search this phase is the observation that the Fermi level touches the van Hove singularity at $t^{\prime} \simeq 0.478 t$ [Fig.1(b)], and the Fermi-surface crosses the Brillouin zone boundary at six van Hove singularity points, i.e., the $M$ points [see Fig.1(c)]. Thus, the particle-hole excitations with transfer momenta $\boldsymbol{Q}_{1,2,3}$ [Fig.1(c)] connecting the van Hove points are the dominant excitations and expected to lead to the emergence of density waves once we turn on the Coulomb interactions. For the on-site Hubbard interaction, the SDW instability will overcome the instability of a charge density wave ${ }^{48-50}$. The spin orientation of SDW order associated with $\boldsymbol{Q}_{1,2,3}$ has the form $\boldsymbol{\eta}_{i}=\frac{1}{\sqrt{3}}\left(\cos \boldsymbol{Q}_{1} \cdot \boldsymbol{r}_{i}, \cos \boldsymbol{Q}_{2} \cdot \boldsymbol{r}_{i}, \cos \boldsymbol{Q}_{3} \cdot \boldsymbol{r}_{i}\right)$, where $\boldsymbol{r}_{i}$ is the site position. Therefore, we can test the existence of SDW order using VCA with the Weiss field $H_{S D W}=h \sum_{i} \boldsymbol{\eta}_{i} \cdot c_{i}^{\dagger} \boldsymbol{\tau} c_{i}$. The results of $\Omega(h)-\Omega(h=0)$ at $t^{\prime} \simeq 0.478 t$ for $U=3,4,5 t$ are shown in Fig.3(a). It can be seen that the SDW order appears only for $U=4 t$ as there is a local minimum. In this way, we can determine its region which is indicated by the red domain in Fig.2. The SDW has the non-coplanar spin order with a 4 -site magnetic unit cell associated with $\boldsymbol{Q}_{1,2,3}$, whose four spin orientations are along the normal directions of the faces of a regular tetrahedron [inset of Fig.3(a)]. The chirality order parameter $\left\langle\boldsymbol{S}_{i} \cdot\left(\boldsymbol{S}_{j} \times \boldsymbol{S}_{k}\right)\right\rangle= \pm 1$ in each triangular plaquette, suggesting the breaking of TR symmetry. However, this scalar chirality is spatially uniform (e.g., positive on all elementary triangles), so the latticerotation symmetry is unbroken. We also note that the chiral SDW order can be constructed in a systematic way in the context of "regular magnetic orders" ${ }^{1}$, based on symmetry considerations.

We further note that when $0<t^{\prime}<\sqrt{3} t$, the $M$ points are always van Hove singularities. They are saddle points of the lower energy band $\varepsilon_{2}(\boldsymbol{k})$ (see Appendix A 1), as can be seen from $\left.\nabla_{\boldsymbol{k}} \varepsilon_{2}\right|_{\boldsymbol{k}=M}=\mathbf{0}$ and $\left.\left[\left(\partial_{k_{1}}^{2} \varepsilon_{2}\right)\left(\partial_{k_{2}}^{2} \varepsilon_{2}\right)-\left(\partial_{k_{1}} \partial_{k_{2}} \varepsilon_{2}\right)^{2}\right]\right|_{\boldsymbol{k}=M}<0$. Therefore, upon doping, the chiral SDW region in the $\left(t^{\prime}, U\right)$ phase diagram will move along the $t^{\prime}$-axis. Specifically, the SDW region moves to larger $t^{\prime}(>0.478 t)$ with hole doping, while towards smaller $t^{\prime}(<0.478 t)$ with electron doping. This opposite trend is due to the absence of particlehole symmetry on a non-bipartite triangular lattice. In addition, the Fermi surface nests perfectly with nesting vectors $\boldsymbol{Q}_{1,2,3}$ for $t^{\prime}=0$ and $3 / 4$ band filling ${ }^{48}$. Therefore, the SDW phase will appear around $t^{\prime}=0$ when the electron doping is around $3 / 4$ band filling.

The metal-NMI transition is determined by the opening of the single-particle gap (Mott gap) via the calculation of spectral function $A(\boldsymbol{k}, \omega)=-\operatorname{Im} G(\boldsymbol{k}, \omega) / \pi$. Usually, the Mott insulator is accompanied by the formation of a magnetic order. In the case of the triangular lattice, the $120^{\circ}$ magnetic order is expected in the large $U$ limit. Therefore, let us test this order by applying Weiss field $H_{A F I}=h \sum_{i} \boldsymbol{e}_{i} \cdot c_{i}^{\dagger} \boldsymbol{\tau} c_{i}$, where $\boldsymbol{e}_{i}=(-\sqrt{3} / 2,-1 / 2,0)$, $(\sqrt{3} / 2,-1 / 2,0)$ or $(0,1,0)$ if $i \in$ sublattice 1,2 or 3 . The result for $\Omega(h)-\Omega(h=0)$ at $t^{\prime}=0$ is shown in Fig.3(b), and local minima suggesting the presence of the magnetic order exist only for $U / t \gtrsim 8$. On the other hand, we find that the Mott gap opens around $U / t=6.7$. Therefore, 
we identify a region $6.7 \lesssim U / t \lesssim 8$ where the correlationdriven insulator exists but without long-range magnetic order. This phase is named as a nonmagnetic insulator (NMI) here and has been suggested as a spin-liquid state $^{38,39}$. With the increase of the Kitaev-like hopping $t^{\prime}$, the $120^{\circ}$ magnetically ordered phase is suppressed, so the region of the NMI phase is extended noticeably, as shown in Fig.2.
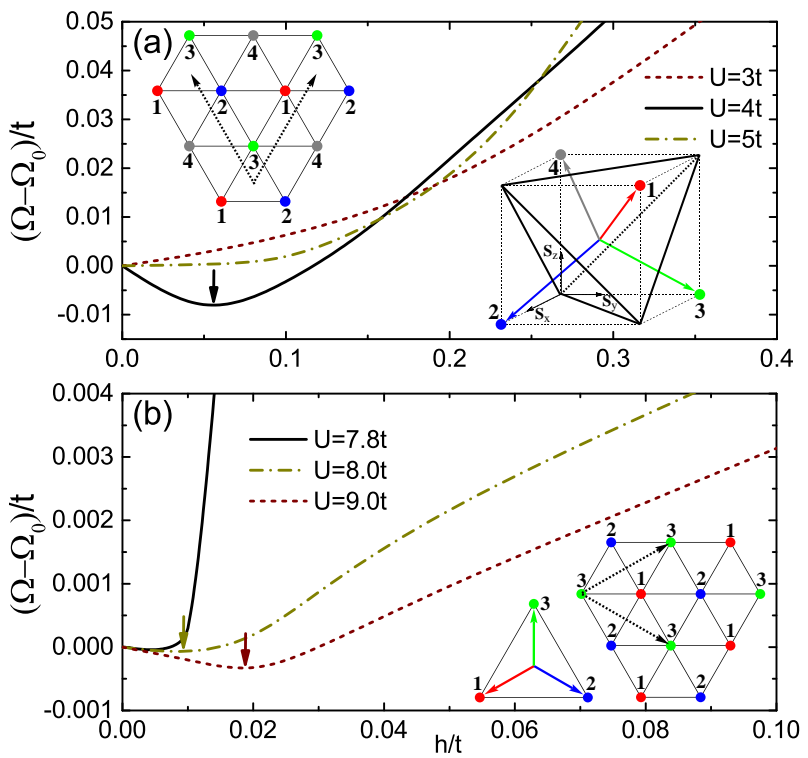

FIG. 3: (color online). Grand potential $\Omega$ as a function of the Weiss field $h$ for (a) the non-coplanar chiral SDW order with a 4-site magnetic unit cell (the inset) at $t^{\prime} \simeq 0.478 t$ and (b) the $120^{\circ}$ Neel order with a 3-site magnetic unit cell (the inset) at $t^{\prime}=0$. The local minima are indicated by arrows. The dashed arrows in the insets represent the magnetic translation vectors.

Let us now proceed to the discussion of interaction effects on the phase with nontrivial topology. As noted above, in the $U=0$ limit, there is a transition from metal $\left(t^{\prime}<\sqrt{3} t\right)$ to CI $\left(t^{\prime}>\sqrt{3} t\right)$ due to the gap opening which arises from the split of energy band by the Kitaev-like hopping $t^{\prime}$. This is an indirect gap as shown in Fig.4(a), where the bottom of the upper band is at the $\Gamma$ point while the top of the lower band is at the $K$ point. With the increase of $U$, we find an interesting low-energy spectral weight transfer. A noticeable accumulation of spectral weight occurs around the $K$ point above the Fermi level, where there is no spectral weight at $U=0$. This leads to the formation of additional bands around the $K$ point with increasing $U$. Eventually, a gap closing occurs at the $K$ point for $U \approx 13.5 t$ at $t^{\prime}=1.8 t$ [Fig.4(b)], and it opens again with the further increase of $U$ [Fig.4(c)]. Therefore, we observe the transition from an indirect band gap of the CI to a direct Mott gap of the NMI. We also find that the critical value $t_{c}^{\prime}=\sqrt{3} t$, which separates the metal and CI phases, does not change with $U$, as shown in Fig.2. Before the single-particle gap closes at the $K$ point, our calculation shows that the nonzero
Chern number of the noninteracting CI survives. Thus, we obtain an interacting CI in an extended $U$ region in the phase diagram Fig.2.
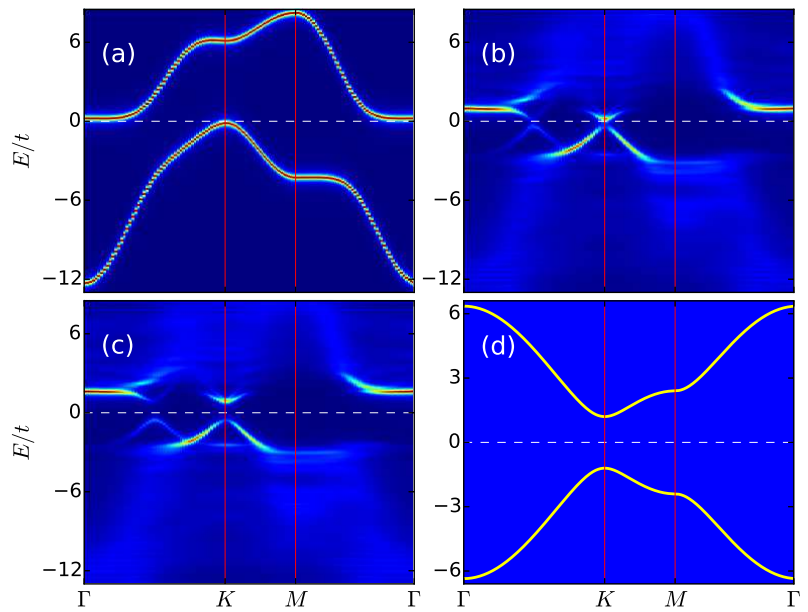

FIG. 4: (color online). (a)-(c) Single-particle spectra along the high-symmetry path in the Brillouin zone computed using CPT, at (a) $U=0$, (b) $U=13.5 t$, and (c) $U=15 t$. (d) The rotor excitation spectrum obtained from the slave-rotor mean-field calculation. $t^{\prime}$ is fixed at $1.8 t$ for (a)-(d).

\section{MEAN-FIELD ANALYSIS}

As an attempt to further understand the NMI phase, we perform the slave-rotor analysis ${ }^{52,53}$. Its reliability in dealing with Hubbard models is supported by more controlled numerical approaches ${ }^{38,52,54,55}$. It has also been suggested ${ }^{2}$ that the slave-rotor method works reasonably well for small to intermediate interactions and magnetically disordered phases near the Mott transition ${ }^{2}$. In this method, one decomposes the electron operator as $c_{i \sigma}=e^{i \theta_{i}} f_{i \sigma}$, with the charged bosonic rotor operator $\theta_{i}$ and the electrically neutral fermionic spinon operator $f_{i \sigma}$. After introducing the mean-field parameters $Q_{f}=\left\langle e^{-i\left(\theta_{i}-\theta_{j}\right)}\right\rangle$ and $Q_{\theta}=\left\langle f_{i}^{\dagger}\left(t \tau^{0}+t^{\prime} \tau^{\alpha}\right) f_{j}\right\rangle$, we can decouple the Hamiltonian Eq.(1) into the spinon and rotor sectors as: $H_{M F}=H_{f}+H_{\theta}$. The spinon Hamiltonian $H_{f}$ is identical to that for the free electron $H_{0}$ except that the band width is renormalized with the factor $Q_{f}$, and the Hubbard interaction term enters the rotor Hamiltonian $H_{\theta}$ only (see Appendix B 2). After performing a selfconsistent calculation, we get the metal(CI)-NMI transition line via the opening of the rotor gap. Before the opening of the rotor gap which corresponds to the weakly interacting regime, the rotors condense. The electron and spinon operators are thus proportional, and they have identical Hamiltonians up to a renormalized factor $Q_{f}$. Therefore, we get the metal and CI phases with $t^{\prime}=\sqrt{3} t$ as the transition line between them. In the strongly interacting regime, the rotor excitations are gapped and become uncondensed, corresponding to the NMI phase ob- 
tained above. In this case, the electron Green's function is the convolution of spinon and rotor Green's functions. For $t^{\prime}<\sqrt{3} t$ where the spinon spectrum has no gap, we get the nonmagnetic gapless Mott insulator (GMI) with a spinon Fermi surface. For $t^{\prime}>\sqrt{3} t$, there is a bulk gap in the spinon spectrum and the spinons have nontrivial band topology with Chern number \pm 2 . This is a fractionalized CI with two spinon chiral edge states.

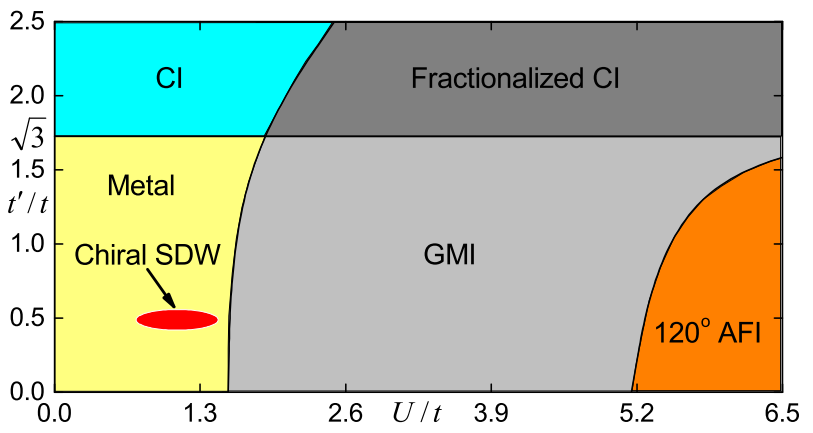

FIG. 5: (color online). Mean-field phase diagram obtained via the slave-rotor and Hartree-Fock approximations. It consists of a metal phase, a Chern insulator (CI), a gapless Mott insulator (GMI), a fractionalized CI, a chiral spin density wave (SDW), and a $120^{\circ}$ antiferromagnetic insulator (AFI).

To treat the interaction-driven magnetically ordered phases at the mean-field level, we express the Hubbard term using spin operators $\boldsymbol{S}_{i}$, and decouple it via $\boldsymbol{S}_{i}^{2} \rightarrow 2\left\langle\boldsymbol{S}_{i}\right\rangle \cdot \boldsymbol{S}_{i}-\left\langle\boldsymbol{S}_{i}\right\rangle^{2}$ (see Appendix B 1). Then, a selfconsistent calculation gives the chiral SDW region and the phase boundary of the $120^{\circ}$ Neel order according to $m \neq 0\left(m\right.$ the magnitude of $\left.\left\langle\boldsymbol{S}_{i}\right\rangle\right)$.

With above results, we obtain the mean-field phase diagram Fig.5. Comparing it with the VCA phase diagram Fig.2, we find a qualitative consistency. From this comparison, we further suggest that the NMI-I and -II shown in Fig.2 would correspond to the GMI and fractionalized CI phases shown in Fig.5, respectively. Based on the slave-rotor analysis, we can also present an understanding of the transition from the indirect band gap to the direct Mott gap shown in Fig.4(a)-(c). In Fig.4(d), we plot the rotor excitation spectrum. Its low-energy spectrum abound the $K$ point shares a similarity with the VCA result presented in Fig.4(c) and shows a direct gap at the $K$ point. From the mean-field analysis, the transition from CI to fractionalized CI is characterized by the opening of the rotor gap, so it is expected that the low-energy spectrum is dominated by rotor excitations.

\section{SUMMARY AND DISCUSSION}

In summary, we have mapped out the phase diagram of the half-filled triangular lattice Kitaev-Hubbard model using the variational cluster approach. It contains a noncoplanar chiral magnetic order, an extended nonmagnetic insulating phase, and an interacting Chern insu- lator. The nonmagnetic insulator has been further classified into a gapless Mott insulator and a fractionalized Chern insulator, based on the slave-rotor mean-field theory.

The gapless Mott insulator is a $U(1)$ spin liquid state with a spinon Fermi surface, which in general has a low temperature specific heat $C_{v} \sim T^{2 / 3}$ ( $T$ the temperature $)^{55}$. It is compatible with the experimental observation of $C_{v}$ in $\mathrm{YbMgGaO}_{4}{ }^{29}$, suggesting that the above gapless Mott insulator (for $0<t^{\prime}<\sqrt{3} t$ ) is a promising spin liquid candidate for the spin-orbit coupled insulator $\mathrm{YbMgGaO}_{4}{ }^{30-32}$. The fractionalized Chern insulator breaks time-reversal symmetry and has a nontrivial band topology of spinons. So it is probably a chiral spin liquid with nontrivial topological order. Recently, a chiral spin liquid with topological degeneracy and anyon excitations was identified in the Haldane-Hubbard Mott insulator ${ }^{56}$. It is interesting to note that some common ideas exist between Ref. [56] and our work: (1) the noninteracting band structure has nonzero Chern numbers; and (2) strong interactions together with frustration may lead to a chiral spin liquid with nontrivial topological order. In the limit $t^{\prime}=0$, Eq.(1) reduces to the usual triangular lattice Hubbard model. Experimentally, it has been shown that the triangular organic materials $\kappa$ $(\mathrm{BEDT}-\mathrm{TTF})_{2} \mathrm{Cu}_{2}(\mathrm{CN})_{3}{ }^{57}$ and $\mathrm{EtMe}_{3} \mathrm{Sb}\left[\mathrm{Pd}(\mathrm{dmit})_{2}\right]_{2}{ }^{58}$ exhibit spin liquid behaviors. To describe these experimental facts, the theories of spinon Fermi surface ${ }^{55,59}$ and of quadratic band touching of spinons ${ }^{60}$ have been proposed. These two spin liquid states have competitive energies and which is more stable depends on the relative strength of model parameters. Our result of the gapless Mott insulator (at $t^{\prime}=0$ ) is consistent with the theory of spinon Fermi surface ${ }^{55,59}$.

Given the rich phase diagram at half-filling, it is interesting to consider possible new phases when the model is doped. One natural consideration is the superconducting states arising from doping a Mott insulator ${ }^{7}$. Let us first discuss the effect of doping the $120^{\circ}$ Neel order. The large- $U$ effective spin model of Eq.(1) is the Kitaev-Heisenberg model in which both the Kitaev and Heisenberg interactions are antiferromagnetic (AFM). In Ref. [28], it has been shown that both the AFM Kitaev and AFM Heisenberg interactions favor a $d+i d$-wave superconductivity (SC) upon doping. We thus expect that the $120^{\circ} \mathrm{Neel}$ order would become a $d+i d$-wave SC under doping. For the intermediate- $U$ NMI phase, it is instructive to understand the doping effect from Anderson's idea ${ }^{61}$ of the resonating-valence-bond (RVB) state and SC: The preexisting spinon singlet pairs in the undoped RVB state become superconducting Cooper pairs under doping. At $t^{\prime}=0$, the NMI phase is a RVB spin liquid probably with $d+i d$ pairing pattern ${ }^{60}$, and a singlet $d+i d$-wave $\mathrm{SC}$ is thus expected upon doping. The NMI phase at nonzero $t^{\prime}$ should break the $S U(2)$ spinrotation symmetry due to the spin-dependent Kitaev-like hopping, which entangles the spin and spatial degrees of freedom like a spin-orbit coupling. Thus, triplet SCs 
(e.g., a $p+i p$-wave $\mathrm{SC}$ ) or their coexistence with singlet SCs probably appear upon doping the NMI phase at nonzero $t^{\prime}$. This is also reminiscent of the honeycomb Kitaev spin liquid ${ }^{20}$ : Its quadratic fermionic Hamiltonian takes the $p$-wave pairing form, and triplet $p$-wave SCs appear upon doping the Kitaev model ${ }^{62}$.

\section{Acknowledgments}

We would like to thank Meng Cheng, Zhong Wang and Jia-Wei Mei for valuable discussions. This work was supported by the National Natural Science Foundation of China (11190023, 11374138 and 11204125).

\section{Appendix A: THE NONINTERACTING limit}

In this appendix, we focus on the noninteracting band structure and its nontrivial band topology.

\section{Band structure}

To obtain the band structure of the noninteracting Hamiltonian $H_{0}$ in Eq.(1), we write it in momentum space as $H_{0}=\sum_{\boldsymbol{k}}\left(c_{\boldsymbol{k} \uparrow}^{\dagger}, c_{\boldsymbol{k} \downarrow}^{\dagger}\right) H_{\boldsymbol{k}}\left(c_{\boldsymbol{k} \uparrow}, c_{\boldsymbol{k} \downarrow}\right)^{T}$ and

$$
H_{k}=\left(\begin{array}{cc}
-2 t g_{k}-2 t^{\prime} A_{k} & -2 t^{\prime} B_{k} \\
-2 t^{\prime} B_{k}^{*} & -2 t g_{k}+2 t^{\prime} A_{k}
\end{array}\right) \text {, }
$$

where $g_{\boldsymbol{k}}=\cos \boldsymbol{k} \cdot \boldsymbol{a}_{1}+\cos \boldsymbol{k} \cdot \boldsymbol{a}_{2}+\cos \boldsymbol{k} \cdot\left(\boldsymbol{a}_{1}+\boldsymbol{a}_{2}\right), A_{\boldsymbol{k}}=$ $\cos \boldsymbol{k} \cdot\left(\boldsymbol{a}_{1}+\boldsymbol{a}_{2}\right)$, and $B_{\boldsymbol{k}}=\cos \boldsymbol{k} \cdot \boldsymbol{a}_{1}-i \cos \boldsymbol{k} \cdot \boldsymbol{a}_{2}$. Diagonalizing $H_{\boldsymbol{k}}$ then gives the energy spectra $\varepsilon_{1}(\boldsymbol{k})=-2 t g_{\boldsymbol{k}}+$ $2 t^{\prime} \sqrt{A_{\boldsymbol{k}}^{2}+\left|B_{\boldsymbol{k}}\right|^{2}}$ and $\varepsilon_{2}(\boldsymbol{k})=-2 t g_{\boldsymbol{k}}-2 t^{\prime} \sqrt{A_{\boldsymbol{k}}^{2}+\left|B_{\boldsymbol{k}}\right|^{2}}$.
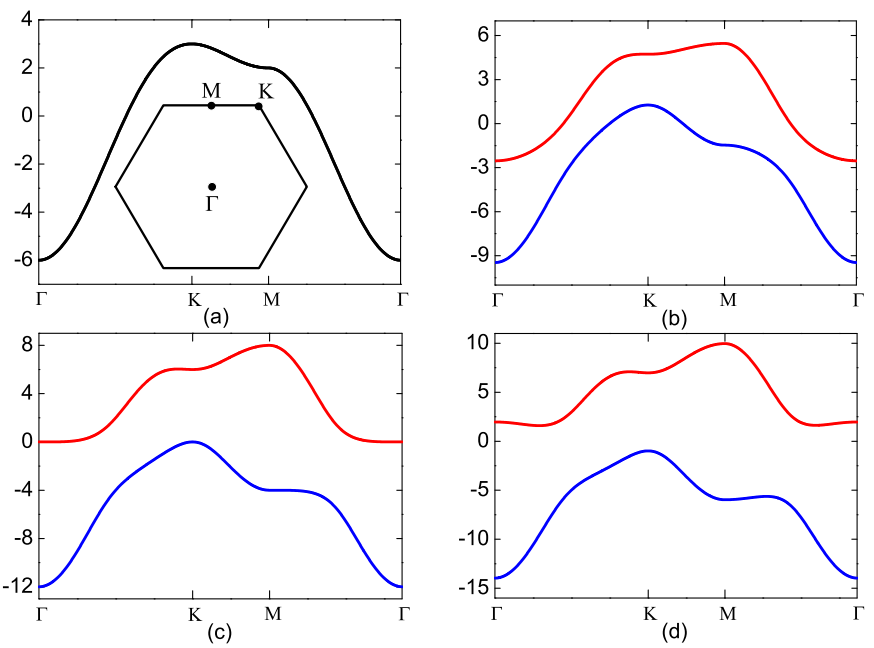

FIG. 6: (color online). Energy dispersion $(t=1)$ of $H_{0}$, along the high-symmetry path in the Brillouin zone [see the inset of (a)], for (a) $t^{\prime}=0$, (b) $t^{\prime}=1$, (c) $t^{\prime}=\sqrt{3}$, and (d) $t^{\prime}=2.3$.
The energy dispersion for different $t^{\prime}$ is shown in Fig.6. As shown, the energy spectrum is split into two distinct bands $\varepsilon_{1}$ and $\varepsilon_{2}$ due to the introduction of $t^{\prime}$. The splitting increases with $t^{\prime}$ and a gap between the two bands appears at $t^{\prime}=\sqrt{3} t$. Therefore, there is a metal-insulator transition at the critical point $t_{c}^{\prime}=\sqrt{3} t$ at half filling. We also notice that the band gap of the insulator is an indirect gap [see, e.g., Fig.6(d)].

\section{Calculation of the Chern number in the noninteracting case}

Since the two energy bands $\varepsilon_{1}$ and $\varepsilon_{2}$ do not touch each other for any nonzero value of $t^{\prime}$, the Chern number $C$ of each band is well defined and can be expressed as the integral of the Berry curvature $b(\boldsymbol{k})$ over the Brillouin zone,

$$
C=\frac{1}{2 \pi} \int_{\mathrm{BZ}} d^{2} k b(\boldsymbol{k})
$$

where $b(\boldsymbol{k})=\nabla_{\boldsymbol{k}} \times i\left\langle\phi_{\boldsymbol{k}} \mid \nabla_{\boldsymbol{k}} \phi_{\boldsymbol{k}}\right\rangle$, and $\phi_{\boldsymbol{k}}$ is the eigenvector of $H_{k}$. A direct calculation of Eq.(A2) shows that $C=$ \pm 2 for each band, and the two bands have an opposite sign in $C$. Thus, the insulating phase at $t^{\prime}>\sqrt{3} t$ is a Chern insulator (CI).

As an illustration to see why the band has the Chern number 2, let us rewrite the $2 \times 2$ Bloch Hamiltonian $H_{k}$ as $H_{\boldsymbol{k}}=-2 t g_{\boldsymbol{k}} \tau^{0}-2 t^{\prime} \operatorname{Re}\left(B_{\boldsymbol{k}}\right) \tau^{x}+2 t^{\prime} \operatorname{Im}\left(B_{\boldsymbol{k}}\right) \tau^{y}-2 t^{\prime} A_{\boldsymbol{k}} \tau^{z}$. The first term $\propto \tau^{0}$ in $H_{k}$ can be ignored because it does not affect the band Chern number. We then smoothly deform the Bloch Hamiltonian by introducing a real parameter $\lambda$ to the last term $\propto \tau^{z}$, say

$$
h_{\boldsymbol{k}}(\lambda)=-2 t^{\prime} \operatorname{Re}\left(B_{\boldsymbol{k}}\right) \tau^{x}+2 t^{\prime} \operatorname{Im}\left(B_{\boldsymbol{k}}\right) \tau^{y}-2 \lambda t^{\prime} A_{\boldsymbol{k}} \tau^{z} .
$$

For any $\lambda>0, h_{k}(\lambda)$ is adiabatically connected to $H_{k}$ and hence they have the same band topology. For a small $\lambda$, we can expand Eq.(A3) around the four Dirac points $(\boldsymbol{K}=(\pi, 0),(\pi, 2 \pi / \sqrt{3}),(0, \pi / \sqrt{3}),(0,-\pi / \sqrt{3}))$ which are obtained at $\lambda=0$,

$$
\begin{aligned}
& h_{1}(\boldsymbol{q})=2 t^{\prime} q_{1} \tau^{x}+2 t^{\prime} q_{2} \tau^{y}+2 \lambda t^{\prime} \tau^{z}, \\
& h_{2}(\boldsymbol{q})=-2 t^{\prime} q_{1} \tau^{x}-2 t^{\prime} q_{2} \tau^{y}+2 \lambda t^{\prime} \tau^{z}, \\
& h_{3}(\boldsymbol{q})=2 t^{\prime} q_{1} \tau^{x}-2 t^{\prime} q_{2} \tau^{y}-2 \lambda t^{\prime} \tau^{z}, \\
& h_{4}(\boldsymbol{q})=-2 t^{\prime} q_{1} \tau^{x}+2 t^{\prime} q_{2} \tau^{y}-2 \lambda t^{\prime} \tau^{z},
\end{aligned}
$$

where $\boldsymbol{q} \equiv \boldsymbol{k}-\boldsymbol{K}, q_{1} \equiv \boldsymbol{q} \cdot \boldsymbol{a}_{1}$, and $q_{2} \equiv \boldsymbol{q} \cdot \boldsymbol{a}_{2}$. When $\lambda=0$, the spectrum of Eq.(A3) becomes gapless at the Dirac point, where the Berry curvature diverges and behaves like a $\pi$ "flux-line", e.g., $b(\boldsymbol{q})= \pm \pi \delta(\boldsymbol{q})$. Therefore, each Dirac point will contribute $\pm 1 / 2$ to the Chern number after turning on $\lambda$. In addition, the four Dirac points have the same contributions (e.g., they are all positive, see Fig.7) due to the same chiralities, and it gives rise to $C=4 \times \frac{1}{2}=2$. 


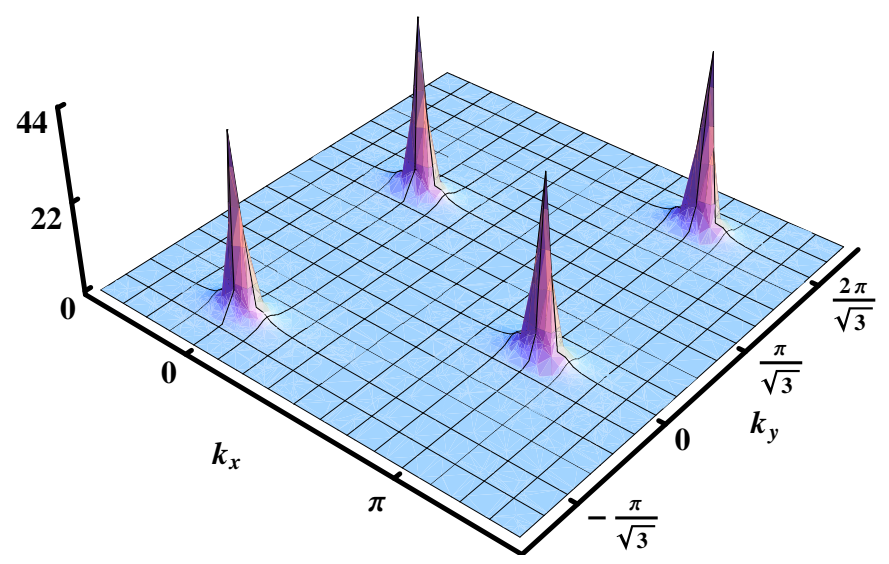

FIG. 7: (color online). Distribution of the Berry curvature in Brillouin zone, obtained from Eq.(A3) for $t^{\prime}=1$ and $\lambda=0.1$. The four Dirac points are indicated by the peaks.

\section{A constraint on the value of Chern number}

Here, we point out a general constraint on the value of the band Chern number for a class of Bloch Hamiltonians $H(\boldsymbol{k})$ in two spatial dimensions, where $H(\boldsymbol{k})$ is defined as a general $n \times n$ Hermitian matrix with $n$ distinct and nondegenerate energy bands $\varepsilon_{i}(\boldsymbol{k})(i=1,2, \cdots, n)$.

Our observation states that: If $H(-\boldsymbol{k})=H(\boldsymbol{k})$ holds in the entire Brillouin zone, then the Chern number $C_{i}$ associated with each band $\varepsilon_{i}(\boldsymbol{k})$ is an even integer. [For example, the band Chern number of Eq.(A1) or (A3) is an even integer equal to \pm 2 .]

Proof: Let us consider the Brillouin zone represented by the parallelogram, as shown in Fig.8(a). We now bipartite the Brillouin zone into two halves, say, a left region and a right region separated by the line segment $C D$. The condition $H(-\boldsymbol{k})=H(\boldsymbol{k})$ implies that its eigenvectors satisfy $\phi_{i}(-\boldsymbol{k})=\phi_{i}(\boldsymbol{k})$ (up to an unphysical phase factor) and the corresponding Berry curvatures also satisfy $b_{i}(-\boldsymbol{k})=b_{i}(\boldsymbol{k})$. Thus, the band Chern number defined by Eq.(A2) becomes,

$$
C_{i}=\frac{1}{2 \pi} \int_{\mathrm{L}} b_{i}(\boldsymbol{k})+\frac{1}{2 \pi} \int_{\mathrm{R}} b_{i}(\boldsymbol{k})=2 \times \frac{1}{2 \pi} \int_{\mathrm{L}} b_{i}(\boldsymbol{k}),
$$

where the notation $\int_{L}\left(\int_{R}\right)$ indicates the integral over the left (right) half Brillouin zone. From the point of view of topology, each $\boldsymbol{k}$ point is equivalent to the $-\boldsymbol{k}$ point in the Brillouin zone, due to the relation $\phi_{i}(-\boldsymbol{k})=\phi_{i}(\boldsymbol{k})$. Therefore, the line segment $\Gamma C$ is identical to the segment $\Gamma D$ [see Fig.8(a)] and they can be glued together to a single segment [see Fig.8(b)]. Because of the periodic structure of the Brillouin zone (i.e., a torus), the segments $B C$ and $A D$ are identical and can be glued together. We also note that the segment $E B$ is identical to $F G$ (due to the periodicity) and $F G$ is identical to $E A$ (due to the equivalence between $\boldsymbol{k}$ and $-\boldsymbol{k}$ ), and hence $E B$ and $E A$ can be glued together. Finally, we notice that any two points inside the left half Brillouin zone are

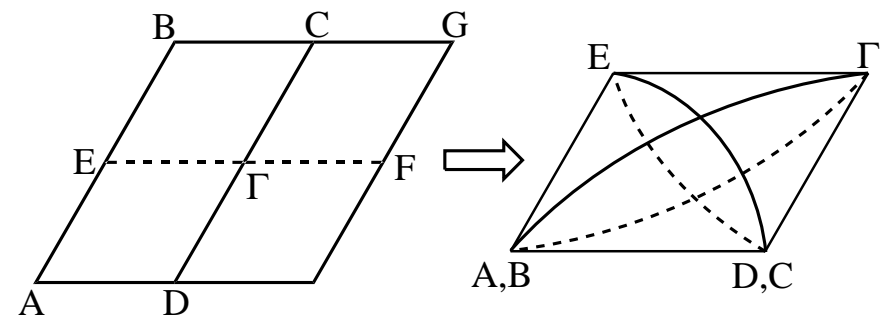

(a)

(b)

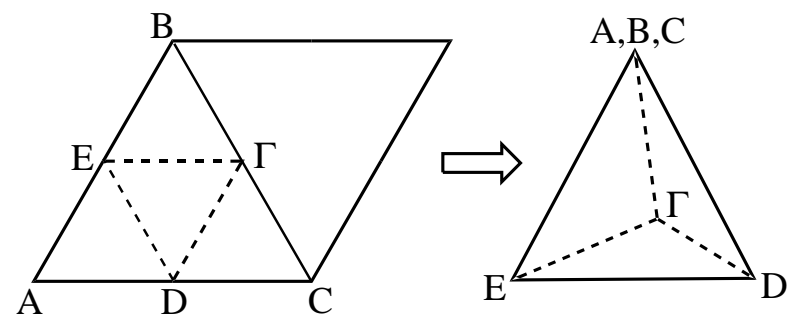

(c)

(d)

FIG. 8: (a)Brillouin zone is represented by a parallelogram, where the $\Gamma$ point denotes the origin, and the points $C, D, E, F$ are the middle points of the corresponding boundaries. (b) The two dimensional sphere which is topologically equivalent to the left half Brillouin zone $A B C D$. (c) Another way to bipartite the Brillouin zone. (d) The tetrahedron which is topologically equivalent to the left half Brillouin zone $A B C$.

distinct. Consequently, the left half Brillouin zone becomes a sphere which is a closed surface [Fig.8(b)]. The upper left region enclosed by $B E \Gamma C B$ corresponds to the northern hemisphere and the lower left region enclosed by $A E \Gamma D A$ corresponds to the southern hemisphere. Mathematically, the integral of Berry curvature over any closed surface must be an integer, which means that the integral $\frac{1}{2 \pi} \int_{\mathrm{L}} b_{i}(\boldsymbol{k})$ in Eq.(A5) is an integer. Eventually, we see that the band Chern number $C_{i}$ in Eq.(A5) should be an even integer.

Remark: The above proof does not depend on the way to bipartite the Brillouin zone. For example, we could divide the Brillouin zone into two halves as shown in Fig.8(c). It can be then shown that the left half Brillouin zone $A B C$ is equivalent to the tetrahedron in Fig.8(d), which is also topologically equivalent to a closed sphere.

\section{Appendix B: Mean-field approach to the interacting case}

Here, we provide details concerning the mean-field (MF) approach to the triangular lattice Kitaev-Hubbard model. This method has been used to give the MF phase diagram (Fig.5) and to further elaborate the nonmagnetic insulating phase obtained via CPT and VCA. 


\section{Magnetically ordered phases}

As discussed in the main text, the chiral SDW phase appears as a weak-coupling instability due to the van Hove singularities. While the $120^{\circ}$ Neel order is stabilized for large $U$. To treat these magnetically ordered phases at the mean-field level, we first rewrite the Hubbard interaction as

$$
U \sum_{i} \hat{n}_{i \uparrow} \hat{n}_{i \downarrow}=-\frac{2 U}{3} \sum_{i} S_{i}^{2}+\frac{U}{2} \sum_{i}\left(\hat{n}_{i \uparrow}+\hat{n}_{i \downarrow}\right),
$$

where $S_{i}^{\alpha}=\frac{1}{2} c_{i}^{\dagger} \tau^{\alpha} c_{i}$. We then decouple Eq.(B1) according to $\boldsymbol{S}_{i}^{2} \rightarrow 2\left\langle\boldsymbol{S}_{i}\right\rangle \cdot \boldsymbol{S}_{i}-\left\langle\boldsymbol{S}_{i}\right\rangle^{2}$. For the chiral SDW phase, the 4 -sublattice order parameters are given by [see the inset of Fig.3(a)]: $\left\langle\boldsymbol{S}_{1}\right\rangle=\frac{m}{\sqrt{3}}(1,1,1)$, $\left\langle\boldsymbol{S}_{2}\right\rangle=\frac{m}{\sqrt{3}}(1,-1,-1),\left\langle\boldsymbol{S}_{3}\right\rangle=\frac{m}{\sqrt{3}}(-1,1,-1)$, and $\left\langle\boldsymbol{S}_{4}\right\rangle=$ $\frac{m}{\sqrt{3}}(-1,-1,1)$. For the $120^{\circ}$ Neel order, the 3 -sublattice order parameters are given by: $\left\langle\boldsymbol{S}_{1}\right\rangle=m\left(-\frac{\sqrt{3}}{2},-\frac{1}{2}, 0\right)$, $\left\langle\boldsymbol{S}_{2}\right\rangle=m\left(\frac{\sqrt{3}}{2},-\frac{1}{2}, 0\right)$, and $\left\langle\boldsymbol{S}_{3}\right\rangle=m(0,1,0)$. Here $m$ represents the magnitude of the magnetization. After the MF decoupling, the quadratic Hamiltonian can be diagonalized and $m$ is calculated self consistently. And we obtain the chiral SDW region and the phase boundary of the $120^{\circ}$ Neel order according to $m \neq 0$, as shown in Fig.5.

\section{Slave-rotor approach}

At the weak and intermediate $U$ case, we apply the slave-rotor MF theory to find the Mott transition and elaborate the possible nature of the nonmagnetic insulating phases. Within this approach, we decompose the electron operator as $c_{i \sigma}=e^{\mathbf{i} \theta_{i}} f_{i \sigma}$, where $\theta$ is the charged, spinless, bosonic rotor and $f_{\sigma}$ the electrically neutral, spinful, fermionic spinon operators. The unitary operator $e^{i \theta_{i}}$ raises the integer rotor angular-momentum quantum number $L_{i}=-i \partial_{\theta_{i}}$ which corresponds to the electric charge. A constraint $L_{i}+\sum_{\sigma} f_{i \sigma}^{\dagger} f_{i \sigma}=1$ should be imposed to restrict the physical Hilbert space of electrons. At half filling, the Hubbard term $U \sum_{i} \hat{n}_{i \uparrow} \hat{n}_{i \downarrow}$ in Eq.(1) can be rewritten as $\frac{U}{2} \sum_{i}\left(\sum_{\sigma} \hat{n}_{i \sigma}-1\right)^{2}$. Thus in the slave-rotor representation, the original Hamiltonian (1) becomes,

$$
\begin{aligned}
H= & -\sum_{\langle i, j\rangle} e^{-\mathbf{i}\left(\theta_{i}-\theta_{j}\right)} f_{i}^{\dagger}\left(t \tau^{0}+t^{\prime} \tau^{\alpha}\right) f_{j}-\mu \sum_{i, \sigma} \hat{n}_{i \sigma}^{f} \\
& +\frac{U}{2} \sum_{i} L_{i}^{2}+h \sum_{i}\left(\sum_{\sigma} \hat{n}_{i \sigma}^{f}+L_{i}-1\right),
\end{aligned}
$$

where we have used the constraint and the identity $\hat{n}_{i \sigma}=\hat{n}_{i \sigma}^{f} \equiv f_{i \sigma}^{\dagger} f_{i \sigma}$. The site-independent $h$ is the Lagrangian multiplier imposing the constraint which is treated on average. In Eq.(B2) every term is quadratic except the hopping term. We can further decompose it as $e^{-\mathbf{i}\left(\theta_{i}-\theta_{j}\right)} f_{i}^{\dagger}\left(t \tau^{0}+t^{\prime} \tau^{\alpha}\right) f_{j} \approx Q_{f} f_{i}^{\dagger}\left(t \tau^{0}+t^{\prime} \tau^{\alpha}\right) f_{j}+$ $e^{-\mathbf{i}\left(\theta_{i}-\theta_{j}\right)} Q_{\theta}-Q_{f} Q_{\theta}$ with the uniform mean-field ansatz $Q_{f}=\left\langle e^{-\mathbf{i}\left(\theta_{i}-\theta_{j}\right)}\right\rangle$ and $Q_{\theta}=\left\langle f_{i}^{\dagger}\left(t \tau^{0}+t^{\prime} \tau^{\alpha}\right) f_{j}\right\rangle$. This reduces Eq.(B2) to two decoupled Hamiltonians for spinons and rotors $H_{\mathrm{MF}}=H_{f}+H_{\theta}+6 N Q_{f} Q_{\theta}-N h(N=$ number of sites),

$$
\begin{aligned}
& H_{f}=-Q_{f} \sum_{\langle i, j\rangle} f_{i}^{\dagger}\left(t \tau^{0}+t^{\prime} \tau^{\alpha}\right) f_{j}+(h-\mu) \sum_{i, \sigma} \hat{n}_{i \sigma}^{f}, \\
& H_{\theta}=-Q_{\theta} \sum_{\langle i, j\rangle} e^{-\mathbf{i}\left(\theta_{i}-\theta_{j}\right)}+\frac{U}{2} \sum_{i} L_{i}^{2}+h \sum_{i} L_{i} .
\end{aligned}
$$

At this stage, the spinon and rotor sectors can be solved almost independently, with their coupling only through the self-consistency requirements on $Q_{f}$ and $Q_{\theta}$.

The spinon Hamiltonian $H_{f}$ has the same form as the free electron Hamiltonian $H_{0}$ in Eq.(1), and the effect of the interaction is to renormalize its bandwidth with the factor $Q_{f}$. In the rotor (charge) sector, $H_{\theta}$ corresponds to the quantum rotor model, which becomes explicit as $Q_{\theta} e^{-\mathbf{i}\left(\theta_{i}-\theta_{j}\right)}+H . c .=2 Q_{\theta} \cos \left(\theta_{i}-\theta_{j}\right)$. At half filling, $\left\langle\sum_{\sigma} f_{i \sigma}^{\dagger} f_{i \sigma}\right\rangle=1$ and hence $\left\langle L_{i}\right\rangle=0$. To satisfy this condition, we take $h=0$ hereinafter, because the external field $h$ coupled to the total angular momentum $h \sum_{i} L_{i}$ breaks the particle-hole symmetry (e.g., $L_{i} \rightarrow-L_{i}$ ) and leads to $\left\langle L_{i}\right\rangle \neq 0$. In the boson picture, we see that $H_{\theta}$ is quite similar to the boson Hubbard model.

The rotor Hamiltonian $H_{\theta}$ contains non-quadratic terms in $\theta$, say $e^{-\mathbf{i}\left(\theta_{i}-\theta_{j}\right)}$, and is hard to solve. We therefore follow Florens and Georges ${ }^{52}$ to replace $e^{\mathbf{i} \theta_{i}}$ by the bosonic variable $X_{i}$ with a constraint $\left|X_{i}\right|^{2}=1$ which is imposed by a Lagrangian multiplier $\rho$. The rotor Hamiltonian then becomes quadratic,

$$
H_{\theta}=-Q_{\theta} \sum_{\langle i, j\rangle} X_{i}^{*} X_{j}+\frac{U}{2} \sum_{i} L_{i}^{2}+\rho \sum_{i}\left(\left|X_{i}\right|^{2}-1\right)
$$

The corresponding action is $S_{\theta}=\int_{0}^{\beta} d \tau \mathcal{L}_{\theta}$. Using the Legendre transformation, we have $\mathcal{L}_{\theta}=-\sum_{i} L_{i}\left(i \partial_{\tau} \theta_{i}\right)+$ $H_{\theta}$ with $i \partial_{\tau} \theta_{i}=\frac{\partial H_{\theta}}{\partial L_{i}}$ which gives $L_{i}=i \partial_{\tau} \theta_{i} / U$. Considering the replacement $X_{i}=e^{i \theta_{i}}$, we have $L_{i}^{2}=$ $\left(\partial_{\tau} X_{i}\right)^{*} \partial_{\tau} X_{i} / U^{2}$. Then, we obtain,

$$
\mathcal{L}_{\theta}=-\frac{1}{2 U} \sum_{i}\left(\partial_{\tau} X_{i}\right)^{*} \partial_{\tau} X_{i}-Q_{\theta} \sum_{\langle i, j\rangle} X_{i}^{*} X_{j}+\rho \sum_{i}\left|X_{i}\right|^{2} .
$$

Now the rotor spectrum can be obtained via the Fourier transformation, which yields

$$
S_{\theta}=\sum_{\boldsymbol{k}, n} X^{*}\left(\boldsymbol{k}, \omega_{n}\right)\left(-\frac{\omega_{n}^{2}}{2 U}+\rho-2 Q_{\theta} g_{\boldsymbol{k}}\right) X\left(\boldsymbol{k}, \omega_{n}\right)
$$

where $\omega_{n}=2 n \pi / \beta$ is the bosonic Matsubara frequency. The energy dispersion of rotors then reads $\xi_{\theta}(\boldsymbol{k})=$ $\pm \sqrt{2 U\left(\rho-2 Q_{\theta} g_{\boldsymbol{k}}\right)}$. 
After solving the spinon and rotor Hamiltonians, the parameters $Q_{f}, Q_{\theta}$, together with $\mu$ and $\rho$ can then be calculated self-consistently via the equations,

$$
\begin{aligned}
Q_{f} & =\frac{U}{3 N} \sum_{\boldsymbol{k}} \frac{g_{\boldsymbol{k}}}{\left|\xi_{\theta}(\boldsymbol{k})\right|} \\
Q_{\theta} & =-\frac{1}{6 N Q_{f}}\left\{\sum_{\boldsymbol{k}^{\prime}}\left[\xi_{1 f}\left(\boldsymbol{k}^{\prime}\right)+\mu\right]+\sum_{\boldsymbol{k}^{\prime \prime}}\left[\xi_{2 f}\left(\boldsymbol{k}^{\prime \prime}\right)+\mu\right]\right\} \\
1 & =\frac{1}{N}\left(\sum_{\boldsymbol{k}^{\prime}} 1+\sum_{\boldsymbol{k}^{\prime \prime}} 1\right) \\
1 & =\frac{U}{N} \sum_{\boldsymbol{k}} \frac{1}{\left|\xi_{\theta}(\boldsymbol{k})\right|}
\end{aligned}
$$

where the relations $n=-\partial F / \partial \mu$ and $\partial F / \partial \rho=0$ with $n$ the occupation number of electrons and $F$ the free energy have been used. $\xi_{1,2 f}(\boldsymbol{k})=-2 t Q_{f} g_{\boldsymbol{k}}-\mu \pm$ $2 t^{\prime} Q_{f} \sqrt{A_{k}^{2}+\left|B_{k}\right|^{2}}$ are the spinon dispersions obtained from $H_{f}$ in Eq.(B3), and the notation $\boldsymbol{k}^{\prime}\left(\boldsymbol{k}^{\prime \prime}\right)$ indicates that only the $\boldsymbol{k}^{\prime}\left(\boldsymbol{k}^{\prime \prime}\right)$ that satisfy $\xi_{1 f}\left(\boldsymbol{k}^{\prime}\right)<0$ $\left(\xi_{2 f}\left(\boldsymbol{k}^{\prime \prime}\right)<0\right)$ are included in the summation. When the energy gap of rotors $\Delta_{g}=2 \min \left(\left|\xi_{\theta}(\boldsymbol{k})\right|\right)$ closes, the rotor bosons condense. The transition to the Mott phase is characterized by the change of $\Delta_{g}$ from zero to nonzero where the rotor bosons become uncondensed. The numerical result of this transition is shown in the MF phase diagram (Fig.5).
* jxli@nju.edu.cn

1 W. Witczak-Krempa, G. Chen, Y. B. Kim, and L. Balents, Annu. Rev. Condens. Matter Phys. 5, 57 (2014), URL http://dx.doi.org/10.1146/ annurev-conmatphys-020911-125138.

2 D. Pesin and L. Balents, Nat. Phys. 6, 376 (2010), URL http://www . nature.com/nphys/journal/v6/n5/full/ nphys1606.html.

3 C. L. Kane and E. J. Mele, Phys. Rev. Lett. 95, 146802 (2005), URL http://link.aps.org/doi/10.1103/ PhysRevLett.95.146802.

4 B. A. Bernevig, T. L. Hughes, and S.-C. Zhang, Science 314, 1757 (2006), URL http://science.sciencemag. org/content/314/5806/1757.

5 M. König, S. Wiedmann, C. Brüne, A. Roth, H. Buhmann, L. W. Molenkamp, X.-L. Qi, and S.-C. Zhang, Science 318, 766 (2007), URL http://science.sciencemag.org/ content/318/5851/766.

6 M. Imada, A. Fujimori, and Y. Tokura, Rev. Mod. Phys. 70, 1039 (1998), URL http://link.aps.org/doi/10. 1103/RevModPhys.70.1039.

7 P. A. Lee, N. Nagaosa, and X.-G. Wen, Rev. Mod. Phys. 78, 17 (2006), URL http://link.aps.org/doi/10.1103/ RevModPhys.78.17.

8 H. L. Stormer, D. C. Tsui, and A. C. Gossard, Rev. Mod. Phys. 71, S298 (1999), URL http://link.aps.org/doi/ 10.1103/RevModPhys.71.S298.

9 G. Khaliullin, Prog. Theor. Phys. Suppl. 160, 155 (2005), URL http://ptps.oxfordjournals.org/content/160/ 155. abstract.

10 G. Jackeli and G. Khaliullin, Phys. Rev. Lett. 102, 017205 (2009), URL http://link.aps.org/doi/10.1103/ PhysRevLett.102.017205.

11 J. G. Rau, E. K.-H. Lee, and H.-Y. Kee, Annual Review of Condensed Matter Physics 7, 195 (2016), http://dx.doi.org/10.1146/annurev-conmatphys031115-011319, URL http://dx.doi.org/10.1146/ annurev-conmatphys-031115-011319.

12 S.-L. Yu, X. C. Xie, and J.-X. Li, Phys. Rev. Lett. 107, 010401 (2011), URL http://link.aps.org/doi/10.1103/ PhysRevLett.107.010401.

13 M. Hohenadler, T. C. Lang, and F. F. Assaad, Phys. Rev. Lett. 106, 100403 (2011), URL http://link.aps.org/ doi/10.1103/PhysRevLett.106.100403.

14 S. K. Kim and J. Zang, Phys. Rev. B 92, 205106 (2015),
URL http://link.aps.org/doi/10.1103/PhysRevB.92. 205106.

15 J. c. v. Chaloupka, G. Jackeli, and G. Khaliullin, Phys. Rev. Lett. 110, 097204 (2013), URL http://link.aps. org/doi/10.1103/PhysRevLett.110.097204.

16 G. Jackeli and A. Avella, Phys. Rev. B 92, 184416 (2015), URL http://link.aps.org/doi/10.1103/PhysRevB.92. 184416.

17 A. Catuneanu, J. G. Rau, H.-S. Kim, and H.-Y. Kee, Phys. Rev. B 92, 165108 (2015), URL http://link.aps.org/ doi/10.1103/PhysRevB.92.165108.

18 I. Rousochatzakis, J. Reuther, R. Thomale, S. Rachel, and N. B. Perkins, Phys. Rev. X 5, 041035 (2015), URL http: //link.aps.org/doi/10.1103/PhysRevX.5.041035.

19 K. Shinjo, S. Sota, S. Yunoki, K. Totsuka, and T. Tohyama, arXiv:1512.02334 (2015).

20 A. Kitaev, Ann. Phys. 321, 2 (2006), URL http://www.sciencedirect.com/science/article/ pii/S0003491605002381.

21 X. Liu, T. Berlijn, W.-G. Yin, W. Ku, A. Tsvelik, Y.-J. Kim, H. Gretarsson, Y. Singh, P. Gegenwart, and J. P. Hill, Phys. Rev. B 83, 220403 (2011), URL http://link. aps.org/doi/10.1103/PhysRevB . 83.220403.

22 A. Biffin, R. D. Johnson, I. Kimchi, R. Morris, A. Bombardi, J. G. Analytis, A. Vishwanath, and R. Coldea, Phys. Rev. Lett. 113, 197201 (2014), URL http://link.aps. org/doi/10.1103/PhysRevLett.113.197201.

23 L.-M. Duan, E. Demler, and M. D. Lukin, Phys. Rev. Lett. 91, 090402 (2003), URL http://link.aps.org/doi/10. 1103/PhysRevLett.91.090402.

24 A. Shitade, H. Katsura, J. Kuneš, X.-L. Qi, S.-C. Zhang, and N. Nagaosa, Phys. Rev. Lett. 102, 256403 (2009), URL http://link.aps.org/doi/10.1103/PhysRevLett. 102.256403.

25 S. R. Hassan, P. V. Sriluckshmy, S. K. Goyal, R. Shankar, and D. Sénéchal, Phys. Rev. Lett. 110, 037201 (2013), URL http://link.aps.org/doi/10.1103/PhysRevLett. 110.037201.

26 S. Rachel, M. Laubach, J. Reuther, and R. Thomale, Phys. Rev. Lett. 114, 167201 (2015), URL http://link.aps. org/doi/10.1103/PhysRevLett.114.167201.

27 H. Yao and S. A. Kivelson, Phys. Rev. Lett. 99, 247203 (2007), URL http://link.aps.org/doi/10.1103/ PhysRevLett.99.247203.

${ }^{28}$ K. Li, S.-L. Yu, and J.-X. Li, New J. Phys. 17, 043032 
(2015), URL http://stacks.iop.org/1367-2630/17/i= $4 / a=043032$.

29 Y. Li, G. Chen, W. Tong, L. Pi, J. Liu, Z. Yang, X. Wang, and Q. Zhang, Phys. Rev. Lett. 115, 167203 (2015), URL http://link.aps.org/doi/10.1103/PhysRevLett. 115.167203.

30 Y. L. Li, D. Adroja, P. K. Biswas, P. J. Baker, Q. Zhang, J. Liu, A. A. Tsirlin, P. Gegenwart, and Q. Zhang, arXiv:1607.03298 (2016).

31 Y. Shen, Y.-D. Li, H. W. Wo, Y. Li, S. Shen, B. Pan, Q. Wang, H. C. Walker, P. Steffens, M. Boehm, et al., arXiv:1607.02615 (2016).

32 J. A. M. Paddison, M. Daum, Z. Dun, G. Ehlers, Y. Liu, M. B. Stone, H. Zhou, and M. Mourigal, arXiv:1607.03231 (2016).

33 T. Dey, A. V. Mahajan, P. Khuntia, M. Baenitz, B. Koteswararao, and F. C. Chou, Phys. Rev. B 86, 140405 (2012), URL http://link.aps.org/doi/10.1103/ PhysRevB.86.140405.

34 Y.-D. Li, X. Wang, and G. Chen, Phys. Rev. B 94, 035107 (2016), URL http://link.aps .org/doi/10.1103/ PhysRevB.94.035107.

35 D. Sénéchal, D. Perez, and D. Plouffe, Phys. Rev. B 66, 075129 (2002), URL http://link.aps.org/doi/10.1103/ PhysRevB.66.075129.

36 M. Potthoff, M. Aichhorn, and C. Dahnken, Phys. Rev. Lett. 91, 206402 (2003), URL http://link.aps.org/doi/ 10.1103/PhysRevLett.91.206402.

37 D. Sénéchal, D. Perez, and M. Pioro-Ladrière, Phys. Rev. Lett. 84, 522 (2000), URL http://link.aps.org/doi/10. 1103/PhysRevLett.84.522.

38 P. Sahebsara and D. Sénéchal, Phys. Rev. Lett. 100, 136402 (2008), URL http://link.aps.org/doi/10.1103/ PhysRevLett.100.136402.

39 A. Yamada, Phys. Rev. B 89, 195108 (2014), URL http: //link.aps.org/doi/10.1103/PhysRevB.89.195108.

40 Z.-L. Gu, K. Li, and J.-X. Li, arXiv:1512.05118 (2015).

41 G. Volovik, Phys. Rep. 351, 195 (2001), URL http://www.sciencedirect.com/science/article/ pii/S0370157300001393.

42 Z. Wang, X.-L. Qi, and S.-C. Zhang, Phys. Rev. Lett. 105, 256803 (2010), URL http://link.aps.org/doi/10.1103/ PhysRevLett.105.256803.

43 V. Gurarie, Phys. Rev. B 83, 085426 (2011), URL http: //link.aps.org/doi/10.1103/PhysRevB.83.085426.

44 Z. Wang and S.-C. Zhang, Phys. Rev. X 2, 031008 (2012), URL http://link.aps.org/doi/10.1103/PhysRevX.2. 031008.

45 Z. Wang and B. Yan, Journal of Physics: Condensed Matter 25, 155601 (2013), URL http://stacks .iop.org/ $0953-8984 / 25 / i=15 / a=155601$.
46 S. E. Korshunov, Phys. Rev. B 47, 6165 (1993), URL http: //link.aps.org/doi/10.1103/PhysRevB.47.6165.

47 T. Momoi, K. Kubo, and K. Niki, Phys. Rev. Lett. 79, 2081 (1997), URL http://link.aps.org/doi/10.1103/ PhysRevLett.79.2081.

${ }^{48}$ I. Martin and C. D. Batista, Phys. Rev. Lett. 101, 156402 (2008), URL http://link.aps.org/doi/10.1103/ PhysRevLett.101.156402.

49 S.-L. Yu and J.-X. Li, Phys. Rev. B 85, 144402 (2012), URL http://link.aps.org/doi/10.1103/PhysRevB.85. 144402.

50 W.-S. Wang, Z.-Z. Li, Y.-Y. Xiang, and Q.-H. Wang, Phys. Rev. B 87, 115135 (2013), URL http://link.aps.org/ doi/10.1103/PhysRevB.87.115135.

51 L. Messio, C. Lhuillier, and G. Misguich, Phys. Rev. B 83, 184401 (2011), URL http://link.aps.org/doi/10.1103/ PhysRevB.83.184401.

52 S. Florens and A. Georges, Phys. Rev. B 70, 035114 (2004), URL http://link.aps.org/doi/10.1103/PhysRevB.70. 035114.

53 E. Zhao and A. Paramekanti, Phys. Rev. B 76, 195101 (2007), URL http://link.aps.org/doi/10.1103/ PhysRevB.76.195101.

54 T. Mizusaki and M. Imada, Phys. Rev. B 74, 014421 (2006), URL http://link.aps.org/doi/10.1103/ PhysRevB.74.014421.

55 O. I. Motrunich, Phys. Rev. B 72, 045105 (2005), URL http://link.aps.org/doi/10.1103/PhysRevB.72. 045105.

56 C. Hickey, L. Cincio, Z. Papić, and A. Paramekanti, Phys. Rev. Lett. 116, 137202 (2016), URL http://link.aps. org/doi/10.1103/PhysRevLett.116.137202.

57 Y. Kurosaki, Y. Shimizu, K. Miyagawa, K. Kanoda, and G. Saito, Phys. Rev. Lett. 95, 177001 (2005), URL http: //link.aps.org/doi/10.1103/PhysRevLett.95.177001.

58 T. Itou, A. Oyamada, S. Maegawa, M. Tamura, and R. Kato, Journal of Physics: Condensed Matter 19, 145247 (2007), URL http://stacks.iop.org/0953-8984/19/i= $14 / a=145247$.

59 S.-S. Lee and P. A. Lee, Phys. Rev. Lett. 95, 036403 (2005), URL http://link.aps.org/doi/10.1103/PhysRevLett. 95.036403.

60 R. V. Mishmash, J. R. Garrison, S. Bieri, and C. Xu, Phys. Rev. Lett. 111, 157203 (2013), URL http://link.aps. org/doi/10.1103/PhysRevLett.111.157203.

61 P. W. Anderson, Science 235, 1196 (1987), ISSN 00368075 , 10959203, URL http://www.jstor.org/stable/1698247.

62 D. D. Scherer, M. M. Scherer, G. Khaliullin, C. Honerkamp, and B. Rosenow, Phys. Rev. B 90, 045135 (2014), URL http://link.aps.org/doi/10.1103/PhysRevB.90. 045135. 